\title{
Public and Open Internet of Things for Smart Cities: The SME Perspective
}

\author{
Pekka Okkonen, Jarkko Hyysalo, Ella Peltonen \\ University of Oulu, Oulu, Finland \\ firstname.lastname@oulu.fi
}

\begin{abstract}
Internet of Things technologies and platforms can provide both novel applications and business strategies for the companies of different technological application areas. However, risks for intensive participation in utilizing novel and expensive technologies into their business and products, might be considered risky by small and medium-sized enterprises (SMEs). Thus, the role of the open source platforms and possibility to test them in the small-scale pilot studies, becomes crucial. In this work, we discuss four different SMEs participating in the open and research-driven IoT pilots in the context of the smart cities. We demonstrate the value of the open Internet of Things platforms can provide for small and medium-sized enterprises working in the area of smart cities, as well as challenges we met.

Index Terms-Smart cities, Business, Best practices
\end{abstract}

\section{INTRODUCTION}

Internet of Things (IoT) and its services are increasingly part of our everyday life, ways of working, and business: personal digital assistant, smart home, smart car and the smart environment are reality with the help of mobile computing and the IoT. IoT is everywhere, in every business area, creating new business opportunities to deliver new services. The global IoT market is estimated to grow to $\$ 471.4$ billion by 2020 [7]. More devices will come online, and a number of platforms and services will be built around them.

There is a huge potential in IoT. The expectations is that, in 2020, the global IoT market is mainly based on three subsectors; Smart Cities (26\%), Industrial IoT (24\%) and Connected Health (20\%), soon followed by Smart Homes (14\%), Connected Cars (7\%), Smart Utilities (4\%) and Wearables (3\%) [5]. Public administrations are especially concerned about Smart Cities, as they will change the way public services are offered (traffic management, water distribution, waste management, urban security environmental monitoring etc.).

As our surroundings are equipped with interacting objects (e.g. sensors, devices, appliances) that can transfer complex data, and are mainly connected via proprietary interfaces, it results in a highly complex interconnected ecosystem with great added value and innovative services for the users. To benefit the most of this environment, interoperability and service portability should be striven for [3]. Standard interfaces should be provided in a network independent and vendor independent manner. With interoperability as a driving force at different levels, from single devices and applications to multifunctional systems, it boosts the digitalization. Services could be provided via different technologies if the same APIs are supported and utilized. As long as open, standard data storage formats and communication protocols are utilized, applications and services can be replaced or upgraded and new solutions developed.

There are has several positive features which allow gaining economies of scale as an IoT ecosystem [2]. High number of applications and data resources provide more resources for reuse and provide knowledge. Virtualization of sources allow the data be processed in homogeneous fashion which simplifies data flow. Dynamics in deployment and service execution are achieved through virtualization which allow seamless processing and use of data sources. In addition, there is shorter time-to-market. Growing real-world cognitive intelligence and readily available data sources allow for faster development and deployment of services.

Previous studies have involved larger corporations and their perspectives to the open IoT development [1], but the special case of small and medium-sized enterprises can be still considered understudied. For SMEs, risks of involving themselves into the pilot projects might be increased due to smaller amount of personnel and resources available. However, SMEs are often considered more flexible what comes to adapting new technologies, and they may benefit the innovation power of novel solutions and pilot-type of testing with errors more than larger-scale corporations.

In this paper, we focus on four small enterprises and pilots run in the context of the smart city and urban computing. In addition to the companies, the city of Oulu and research institute partners participated in conducting the pilots. Based on the interviews performed with the participating companies, we present lessons learned and seek answers to the questions if the public open IoT platform can provide value for SME companies and their customers, and what kind of business opportunities there are. We present the following contributions:

- We provide real-world based definitions for the value in the special case of SMEs participating in open IoT platform implementation and utilization.

- We demonstrate how these values can be realized in shortterm pilot studies.

- We provide important lessons learned for the future open IoT platform pilots considering small enterprises.

\section{RELATED WORK}

Enabling IoT Ecosystems Platform Interoperability. The challenge of the existing IoT platforms comes not in the variety of available platforms, but limited interoperability and crosscollaboration. Bröring et al. [3] define the three requirements 
or key pillars for the truly interoperable IoT ecosystem. These are, 1) the common API, 2) well-defined information models, and 3) a marketplace to monetize access to resources. The main challenge is to create a ecosystems that can connect multiple platforms and allow new development of applications that can performs several connected tasks relating, for example, people's daily life through connected platforms.

The European Platform Initiative (IoT EPI) ${ }^{1}$ is an initiative to create a collaborative IoT ecosystem for IoT platforms cross the European countries, however, keeping the small and mediumsized enterprises in the loop of the development requires understanding their special needs for such platforms. Scuotto et al. [11] motivate for open innovation and encouragement for companies to participate in IoT projects with public sector. Open innovation links multiple entities to exchange knowledge, combine different skills, and take advantage of cooperation to generate innovation.

Sharing domain-specific platforms and allowing other to use them to some extent is a good enabler for cross-domain solutions. Soursos et al. [13] discuss how IoT consists of vertical and specialized solutions targeting specific markets. Companies are developing their solutions in a sort of "silos", which causes each solution be differing - while the number of IoT solutions is growing, so is the variety of them. The IoT platforms have been vertically extended to cover required functions and services. This is however time consuming for companies and cross-domain applications would be much more viable option. Instead, Soursos et al.[13] suggest interoperable and more open solutions to provide cross-domain solutions. Sharing resources is important, because it allows companies to distribute individual strengths, reduce costs, and develop stronger technical solutions. The "virtual IoT provider" is a concept supposed to act as a broker between IoT platforms and solutions and provide new kind of solutions to the market. This operation creates new business cases for not only the new brokers but for IoT platform providers and application developers by increasing interoperability.

Commercial companies contributing into open source domain. Benefits that companies receive can versatile rather than being only complimentary services like some earlier research suggests. Andersen et al. [1] provide a case study conducted at three IT companies (Accenture, Arktekk, and Redpill Linpro) to find out the drivers for commercial companies to contribute to open source. They report three main drivers:

1) Selling complementary services. This allows companies to sell complementary products like training, technical support, consultancy, and certifications. In addition, free software has a greater amount of possible clients compared, and open-sourcing can give competitive advantage compared to other commercial products and thus allow more customers to buy complementary products.

2) Building greater innovative capability. Companies can simply value innovations to be freely revealed in order to benefit the common good. Even so, innovation can also

\footnotetext{
${ }^{1}$ http://big-iot.eu/
}

be protected with intellectual property laws which allows open innovation without the risk of someone benefiting from others' work. Open-sourcing can help companies understand customers better through the customers participating the application design. In addition, open-sourcing can be developed faster and can function as a testbed for new functions to the commercial products - it directly allows the company and customers co-create new features to commercial products.

3) Cost reduction through open-sourcing to an external community. In some cases, companies may see the community working as developers for free. In addition, active open-source communities tend to report bugs and work as "testers" for software. In the best case, opensourcing allows companies to test new additions on beta products with the help of community.

Andersen et al. [1] also highlight that the companies have their own motivations to utilize and publish open source code or documentaries, and each company leans different the motivational factors. Out of the studied companies, Accenture is most motivated by open-sourcing and cooperating with open source companies. Arktekk utilizes the innovation power that their open-sourcing generates, which motivates other companies to participate, too. Redpill Linpro is mostly motivated by the complimentary services that can be sold along with opensourced software. Similar findings are reported by Silva et al. [12], who present a business model for the large-scale SmartSantander IoT Testbed network with 12,000 sensors. Our study complements these findings from 2012 and 2014 with newer results and especially focuses on small companies.

Business Models for IoT Platforms. Berkers et al. [2] provide an example of multi-sided business model which consists of four different companies relating to traffic challenges and they combine their forces to create a horizontal IoT service platform. In this paper, multi-sided business model is seen as essential for the platform as it provides more value to companies than they could achieve individually. More companies involved, larger is the supply of information which also feeds the platform's machine learning and makes the platform more efficient. Also more applications can be build with more data sources. Seeding strategy is seen to be good strategy to get the platform going - getting an existing company to provide solutions for their customers and thus showing the functionality and value of the platform. Thus, multi-sided business model allows companies to build new kind of business.

\section{RESEARCH METHODS}

\section{A. Pilots}

The CityIoT project implements an IoT platform ${ }^{2}$ for pilots. Open environment - the data integration platform - enabled utilization of various IoT platforms as interfaces were built as vendor agnostic. The focus area of our pilots was within collaboration between cities and local small and medium-sized companies focusing on the IoT solutions that can be used to

\footnotetext{
${ }^{2}$ http://pan0107.panoulu.net/
} 
build a smart urban environment. The expertise fields of the companies vary from general and technical management of buildings and urban spaces to developing novel IoT-driven solutions in the urban setting.

Pilots were run as "fast trials" adapting the ideas of Ries [10]. The participating cities provided challenges to be solved with innovative applications and services. Companies can easily and quickly get down to challenges that the cities face as well as design and test their products with real users in real environments. Cities, on the other hand, get knowledge on new digital solutions and cost savings that they can provide.

Pilots were three month in length, starting with the selection of themes and planning, followed by the piloting, and finally the evaluation with learning outcomes. Solid ideas were quickly identified and the ones that were not as promising quickly discarded. The selection criteria were innovativeness, scalability, agility and customer orientation. Pilots had three phases:

1) User mapping: Data gathering with, e.g., interviews and observation.

2) Analysis: Observations, good practices, bottlenecks, processes etc. are analysed.

3) Prototyping and collaborative development including workshops with users and customers, and testing and evaluating the prototypes.

Concrete results of the pilots are, including but not limited to, description of service ideas, stakeholder mappings, use cases, service blueprints and concepts, and prototypes.

\section{B. Open IoT Platform}

The selected technology for our IoT platform was Fiwarebased [6]. Fiware offers open, sustainable and royalty free ecosystem. It is based on NGSI v2 (Next Generation Service Interface) standard and implementation oriented software standards. Focus areas being, for example, on breaking down data silos by utilizing standards.

Our Fiware platform consist of components that each have their own responsibilities. Platform is built only with the components that are necessary. Interfaces and data models follow the NGSI v2 standard. The Fiware community offers various extensions and components ${ }^{3}$.

The platform architecture builds upon Fiware I/O standards, data models, data repositories, and security and authentication sources. Interfaces are the key, as they provide the integration points to system and enable data transfer. Utilization of existing standards provide as wide as possible interoperability.

The architecture is not restricted only to these technologies. The core is the data storage that provides data for applications and services. The data storage can be modular or distributed Orion Context Broker registers the stored entities and the latest changes to their attributes as well as offers interfaces to data storing and retrieval. It is also possible to request only the changes to attributes. Orion Context Broker provides NGSI v2 based service paths for distinguishing the data items.

\footnotetext{
${ }^{3}$ See Fiware catalogue: https://www.fiware.org/developers/catalogue/
}

Only the latest history data is stored in Orion, so additional components are necessary for long-term storage. Several alternatives are available, such as:

- STH-Comet, which is MongoDB database utilizing component,

- QuantumLeap that utilizes CrateDB time seriesdata base,

- Cygnus that is based on Apache Flume, and

- Draco, based on Apache Nifi.

Other components are, for example, various IoT agents that help to transfer data to the platform, WireCloud Widgets for data visualization and dashboards, CKAN extensions enabling publication, search, discovery and consumption of data.

\section{Involved Companies}

\begin{tabular}{|c|c|c|c|}
\hline Companies & Employees & Turnover (euros) & Year of establishment \\
\hline Company A & $5-9$ & $0-0,2 \mathrm{M}$ & 2010 \\
\hline Company B & $5-9$ & $0-0,2 \mathrm{M}$ & 2017 \\
\hline Company C & $5-9$ & $0,2-0,4 \mathrm{M}$ & 2006 \\
\hline Company D & $1-4$ & 0-0,2 M & 2006 \\
\hline
\end{tabular}

KEY NUMBERS OF THE INVOLVED COMPANIES.

There are a total of four companies involved in the research who also participated in the pilot. In order to preserve anonymity we will name the companies as Company A, Company B, Company C, and Company D. All the companies function in IoT business and are relatively newcomers in the market. The companies can be described as micro-enterprises since they all have fewer than 10 employees [9]. Differences are found in the actual age of the companies and in the solutions that they offer which are surprisingly different. Total of four persons were interviewed, one person from each of the companies. Roles of the interviewees are given in the company descriptions.

Even though companies are currently micro-enterprises, all of the interviewees estimated that their company is currently in and transitioning into a growth stage. For example, Company $\mathrm{C}$ and Company D had been developing their offering several years and felt that their product/service was now finalized for larger market adoption. Each of the companies had recently acquired new customers and were now on target to grow their business significantly compared to previous years. This means that the companies are on track of becoming small enterprises in the next few years. This was also shown in the answers of the interviewees. The interviewees felt that their companies are not currently ready for business collaboration as their businesses were heavily focused on growing the sales of their core product. However, the interviewees saw the possibility of getting different kind of growth by collaborating with other companies and organizations.

Company $A$ has a history of building 3D visualization solutions which virtually presents for example urban planning. These virtual solutions are meant to help communicate ideas and plans more fluently between different parties. Company's business model is to license their cloud platform which allows building the visualization models. Customers usually vary from 
design agencies to cities but customer can be anyone who wants to communicate their plans to others, in a simple virtual form. Business model has however been changing from building large solutions for individual customers to automating their processes in order to reach larger audience. This will reduce the workload on the company and help get more customers. Also the pricing model has experienced similar change, transforming from costly one-time payment to smaller payments and even monthly payments. Even though the company has been operating for several years, their product, especially the new version is in early phases. So, company describes themselves being in growth phase as they are now focusing on selling their services to new customers. The company CTO was interviewed.

Company $B$ is recently founded company providing complete IoT solution to customers. CTO of the company was interviewed. Company has developed their own sensor for specific purpose and also created a cloud service around the device. Their solution can be sold individual to customers as well as larger company or organization. Pricing model can be fixed price or monthly payment depending on the customer needs. Company has recently finished their product development phase and can be now described as scale-up company. They can be said to be operating in a niche market and the CTO of the company said that they are looking for growth by excelling in their market area.

Company $C$ has also developed their own sensor technology which is in the core of their business. Sensor is created for a specific purpose but can be offered for various different customers. The product is a sensor solution which enables new kind of business which has not been efficient enough in the past. Similar to Company A, Company $\mathrm{C}$ has also been operating for several years but started developing the current product few years ago and is now looking to expand their business. Company $\mathrm{C}$ as well had their CTO participate in the interview. CTO believes that their main product is a information product that is provided by the sensors. Company processes this data and then enriches the data with other data. This enables different kind of analyses for specific purposes and creates high value for customers.

Company $D$ provides an IoT platform solution for customers who have an idea for IoT service but do not have the platform to implement it. The platform is focused on efficient and effective data transfer which the company has been developing several years. Like others, Company D is shifting from product development to growth phase. Company is looking for new customers to get their platform going. In addition to the platform, company offers consultation and does larger projects with customers to help them build their own IoT solutions. The interview was conducted with the CEO.

\section{Interviews}

The research was carried out as an interview-based survey with companies in the area of IoT. This research method can be described as a qualitative as the number of companies involved is quite marginal. Thereby it would be hard to make any analyses statistically or by any other methods of quantification.
Also, qualitative studies are suitable for investigating open research questions which are answered based on opinions and possible relations found between interviews [8]. The questions of the interview are given in Table II.

The interview questions can be divided in three categories which also function as the structure of the results chapter: 1) Data entity, 2) Open IoT platform, and 3) Pilot assessment. In addition, we consider the category 0) Involved Companies which consists of four questions and provides the basic information about the companies. Second category consisting of three questions, Data Entity handles company's data what they use, where do they get it and what kind of data processing is needed to make data valuable. Following is the main category of the interview consisting of six questions, Open IoT Platform which clarifies companies' thoughts and opinions about the public open IoT platform and tries to find collaboration possibilities. Last three questions are under the Pilot category which has diverse questions relating to pilots and what kind of benefits the pilots have to this kind of research.

Furthermore, when assessing the value of something it can be hard to make conclusions based on purely quantitative research. Value can be defined in countless ways and the definition of value depends on who is answering the question and in what kind of situation the question is asked. Value is often associated to business world when talking about the value that companies provide for their customers. [4] Value can however be basically anything, physical or intellectual relating to for example education, hobbies or everyday life. Again, depending on who is the target of the question. Thus, it is logical to address this kind of subject via a qualitative research.

The interviews were held in a relatively short time-period. This means that there was no time for refining the questions. On the other hand, the interview group being so small it would not have been wise to change the questions between interviews. The interviews consisted of two persons, the interviewer and the company representative. They were held on Skype or in person and lasted for about an hour. Every interview was audiorecorded and also notes were taken. The interview sessions started with brief introduction to the topic and the interviewer telling the motivation for the interview. The interviews followed the question frame consistently enhanced with follow-up questions either for clarification or general interest in the topic. The companies are doing relatively different kind of business which means that some of the questions were not perfectly suited to every company. In those cases, the interviewer refined the questions slightly to get suitable answers.

Last part of the process was to analyze the data and make conclusions on the answers. First the notes needed to be supplemented with recordings. After the interviews were transcribed, started the analysis phase. First, each company was analyzed individually taking notes and then comparing the companies among themselves. Comparison of the answers was the main part of this research because the topic being quite open and dependant on opinions. Therefore conclusions could only be made based on comparing the answer and finding either similarities or divergence between the answers. 
1) What are your business model, life cycle stage, and short-term future plans?

2) Can you give price examples from your service/product?

3) What kind of data you use and where do you get it?

4) Do you combine data from different sources and what opportunities you see in data combination?

5) How do you process the data and how does it affect the value and usability of the data?

6) What is your opinion on open source IoT platform that would be managed by the city?

7) Would your business model be suitable for collaboration with public and open IoT platform?

8) What challenges you see in cooperation with open IoT platform?

9) What kind of data and in what extent you would like on the platform?

10) Would you be open to releasing open source version of your applications/tools to the platform?

11) Would you provide data to the platform for free or for a fee?

12) Which sensors were used in the pilot and how they were chosen?

13) How much time did it take to get integrated to the platform and where there any problems maintaining the system?

14) Did the pilot raise any new ideas regarding your product or business model?

TABLE II

INTERVIEW QUESTIONS

\section{RESUlts}

\section{A. Data Entity}

First topic in the interview was different aspects of company data. The type of data that companies use varies greatly. Some of the interviewed companies have their own sensor base, when where as other companies use data that they get from their customers. The interviewed companies use quite versatile data, for example, Company A uses paper blueprints and pictures and transform them into digital form. Company B and Company C use their own kind of sensor data. Company D uses any kind of data on their platform that their customers have. Companies also typically use data from their partners or open-sourced stores, for example, weather data, to enrich their own data.

Along collecting the data, a large part of the companies business is combining the data with other data. Company A's business model is built around combining complex data and visualizing it in an intelligible form. On the contrary, Company B basically only uses their own data collected from their sensors. However, there has already been discussions on starting to combine their data with some other data. Sensor data is also the main data source for Company $\mathrm{C}$ but they combine their data with other data depending on the customer. Data combination can be combining same time period data together or adding some relevant data along with their sensor data. This kind of combination allows Company $\mathrm{C}$ to comprehensive analysis on their data and provide it for their customers. Company D is in favor of data combination but can not provide examples on their data combining because they do not have a specific data or analysis methods that they use. Depending on the customer needs, Company D combines data when necessary.

Answers show that data combination is not necessary for all companies but can provide opportunities to create additional value for customers as well as the company itself. All companies agree that it is profitable to combine data together and even though one company does not do that yet, they have plans for it in the future.

Data combination can mean different things. Basic combination of the actual data to for example surrounding environment is practically always done. Data can be also combined with different datasets or with complete different types of data. This kind of combination might bring problems if the data models differ considerably from each other. Combination should always be technically doable but especially in smaller companies, it may not be worth the effort. Is the reward from combining the datasets enough to cover the costs and time spend to make the software changes? Company should take into account what effects the changes have on their existing platform like increased delays or complex maintenance. Another thing to consider is that if the company sees the combination process beneficial, should they try to do the whole process themselves or make use of partners or existing platform solutions. Here an open source IoT platform, which is capable of combining different kind of data effortlessly can be useful and help especially smaller companies to grow their business.

Before data can be combined or even used it usually needs to be processed in the application-specific way. For Company $\mathrm{A}$ and Company B it is necessary to process and transform the data before it is usable. Company $\mathrm{B}$ uses machine learning to transform information on picture to data format suitable for their IoT platform. Company $\mathrm{C}$ also handles sensor data but they need to first filter the correct readings from the sensor and then transfer that to numeric format. Company $\mathrm{C}$ says that they also analyze the customer needs based on their behaviour and then offer refined data for the customer's needs. Their goal is to combine the processed data with other relevant data and generate so called "information products" that offers increased value to their customers.

After the data has been preprocessed and structured, the data may still need some kind of refinement in order to deliver proper, applicable information out of the data. Company B said they have to define certain ranges and limits for their measured data. This is especially important for customers to easily see meaning of the data. Company A has a different kind of need for data refinement: their service is running on a web browser so they have to "lighten" or otherwise optimize the data in order to run their service smoothly. Similar platform optimization example was given by Company $\mathrm{D}$ but their actions also take advantage of data combination. Company D collects performance data from communications network, where their platform is connected, analyze the data and then use that information to optimize their own platform. They believe that this kind of expertise is what makes the company stand out from competitors. Generally speaking data allows companies to do statistics to help them define their data more precisely or control their platform's functions.

\section{B. Open IoT Platform}

This section handles companies' opinions and thoughts on the presented public open IoT platform and the questions tried to discover what kind of opportunities and challenges would the 
platform provide. General opinion of the platform was highly positive. Companies thought that it would be great if the city had their own platform where would be large amounts of data available for companies and organizations. Large amounts of data could build interest and generate new innovations inside companies. Company B said that the city has a great amount of data but does not benefit anyone unless the data is used wisely. Here the platform could be a good fit since it would provide a change for companies to utilize data for productive purposes. Companies could, with their expertise, build new applications and services from the data, which the city can not make with limited resources. This kind of innovation could benefit the city either directly through the services or indirectly by better employment and taxes.

Some suspicions were presented by Company $\mathrm{C}$ on competition that the platform could cause. However, the competition was not seen as a threat since the platform is seen to operate with a different business model than the company. Company $\mathrm{D}$ took this idea of competition little further and presented a situation where the city platform could create monopoly, which would not be at all desirable. Following this, Company D pondered if the platform would be needed at all. Similar data distribution could be done between by standardizing data formats and using open APIs. In theory, this kind of network could be a good solution. With removing the middleman data transfer should be easier and faster. However, it is hard to imagine that companies would start cooperating without proper mediator and it would be much more complex to integrate every company individually rather than using centralized platform.

Business-wise, the companies also thought their business model would suit in collaboration with the platform. Companies have develop their own platforms for a long time and they thought that it would be easy to integrate their platforms with any other platform. Also the pilots prove that technically there are not that many problems in the integration process. Company A said that many IoT platforms do not provide necessary analysis tools and Company A would fit this need perfectly and could build applications from plain data. Similarly Company $\mathrm{B}$ and Company $\mathrm{C}$ believed that they could benefit from the platform if there were suitable data for them. Company D thought that it would require customer demand before they could and it would be favorable to start building applications.

Even though the platform seems promising and might provide good opportunities for companies, a few challenges or doubts were discussed in interviews. Platform itself could provide challenges depending what kind of platform is chosen. If the IoT platform is open source, are there enough developers to keep the platform running for several years and evolve it continuously. And if the platform is built up by software company it might be challenging for city employees to have enough knowledge of the platform to maintain it properly. This might bring delays to solving technical problems. On the other hand if the maintenance is done by the software company it might be too expensive for city to afford. Other than these, technical problems are not seen as a threat by the companies. "Nowadays technical problems are always solvable”, says Company B.

Some of the largest challenges that companies came up are related to monetary costs of producing and sharing the data. If the data is free who is going to pay from the applications. Company A brought up cities' perspective saying that smaller cities or municipalities might not be willing to give up data for free due to money difficulties. And if the data costs something, who is going to start developing services, especially if there are not certain customers insight. Of course, there are similar challenges when initial investments are needed in order to start a completely new company. Usually, behind every company there is a business idea that justifies the investments. The same analogy works with the data; to begin with, the data on the platform should be highly relevant or there would have to be large amount of it to drive innovation.

Some challenges were also presented relating to data. If the platform would hold large amounts of data from various different sources, who would own the data and how would the right regarding using and selling be distributed. Another question is that is it possible to get enough data on the platform. Companies might not want to hand over valuable data without getting something in return. And does the city have enough resources to collect enough data without the help of companies. Interesting fact relating to data of the city was pointed out by Company A. Company A has done cooperation with the city of Oulu before and said that the data that city has collected for different purposes is not actually owned by the city. Companies who have built the services and applications for city own the data measured in the city. So even though there is a good amount of data measured from the city, it might not be simple to get data on the platform for free.

When asked what kind of data the companies would like to have on the platform there was a dichotomy between companies. Company B wants the same kind of data that they are measuring themselves which they could easily make use for their business. Company $\mathrm{C}$ said that they would preferably want the same kind of data as they are now using. On top of that they could also use some general data like weather and energy consumption data that could be combined with their own data. Company A and Company D agreed that the data is dependant on customer. It might be possible to build services on plain data but it would be hard to come up with solution that customers would need before consulting them first.

Company A raised another point to consider: "It would be utopian to think that someone would just measure data and push it to the platform out of kindness". Altogether it is not that simple for companies to say how much and what kind of data they want on the platform. Answer could be obtained by short experiments similar to pilots in order to get understanding from companies' needs and new ideas for utilization of data.

The last part of the interview covers companies' preparedness to provide data and applications to the open IoT platform. Regarding applications and tools, companies were open for the idea and discussions had been had in few of the companies. However any plans for open sourcing own products have not been made yet. This is understandable because companies are 
at the beginning of their journey and do not have the resources to develop open source versions. Company $\mathrm{C}$ and Company $\mathrm{D}$ also said that they do not actually have any applications to open source because they are the core parts of their business.

Similar thoughts came up when asked on sharing the data they use. Companies were quite open on sharing the data, especially if they would benefit from it in some way. Companies are also open on being the provider or the user of the data. Company $\mathrm{C}$ is already distributing their data forward since they have quite sophisticated data analysis process and they are confident that others can not benefit financially from the data. Also data of Company $\mathrm{C}$ is completely anonymous which makes the data distribution process harmless. With other three and most of the companies measuring some kind of data, there is a large problem when talking about data distribution. Almost all of the data which companies use is owned by the customers. In order to distribute customer data, customer approval is needed. In addition Company B pointed out that even though the customer agrees to the distribution, there might be some legal barriers on distributing or monetizing the customer data.

\section{Pilot Assessment}

Final part of the interview was supposed to clarify briefly what kind of effort is needed to participate in the pilot and what kind of benefits the companies gained. Every company had different kind of sensors for their pilots and there was also divergence on how the sensors were chosen for the pilot. Company B and Company $\mathrm{C}$ having their own sensors, they were the clear choice for the pilot. Sensors were designed to do the exact measurements and performed flawlessly. Company $B$ pointed out that at least for them sensor development is not essential. Instead, the key focus for them is in developing machine learning and analysis on their platform to diversify their services. Company D did not have their own sensors but used sensors which they were familiar already. This was obviously valuable, especially since the sensor was not perfectly designed to measure the needed subject. Company D had to build the appropriate analysis around the sensor data in order to provide the needed results from measurements. For Company A the sensors were already been chosen when they became part of the pilot. As a result the sensors were not correctly chosen and ended up providing wrong measurements. Therefore the service building process was quite hard in the beginning. Luckily there were some other sensors placed in the pilot area and Company A could use the data from them. This data was correct type and good quality and company managed to build everything they were supposed to in the pilot.

Pilots were performed by using the Fiware open source IoT platform [6]. Companies thought that the platform was predominantly good and behaved as needed. Integration process was simple and took only from few hours to couple of days. General opinion was that API met the standards and documentation of the platform was sufficient. Company B said that the data models were slightly complicated but manageable.

Company A had previous experience on the platform and was able to tell some development areas of Fiware platform.
For starters, if the number of sensors were to grow much higher there would be difficulties how to register and store the sensors sensibly. This is due to Fiware does not have any device management system built-in. Also saving data from multiple sources requires somewhat conformity because platform does not have uniform data model or automated checks for the form of data. Instead Fiware has different data models for different purposes which can be difficult to combine into a congruent application. There is also a lack of out of the box visualization tools which can be found on similar open source IoT platforms. Worthy of attention is that companies only pushed data to the Fiware platform. All of the analysis and visualization were done on their own platforms. Therefore excluding Company A's previous experience, companies do not have comprehensive user experience of the platform so the opinions from this interview can only be bound to the integration process.

Everyone thought that the pilot was interesting and raised new ideas because they had not participated in similar projects before. Company A found the pilot truly eye-opening for their business. They had to build a new kind of visualization solution which as such was a tremendous learning process. In addition company used real-time data and visualized an indoor space for the first time. As a result Company A found out a new market space in indoor property visualization and is now in business with several customers in that area. Regarding data the company realized that the blueprints they use for visualization can actually be quite plain which was a significant observation because this reduces their service building time considerably. Some improvements were also discovered relating to data enrichment and what kind of results customers want to see.

Company B gained first time experience in deploying a solution in a large destination. Company learned how data behaves in a larger target and they learned to handle it efficiently. They also came up with interesting idea on how to take advantage of the data measured in the pilot and use it to generate possible saving for the public sector. This shows that these kind of pilots can generate remarkable business ideas which benefits not only the company but others as well like the public sector. For Company $\mathrm{C}$ a new range of use for their sensors was out which might be a new business area as well. Company will test this new area in a new pilot during this fall. In addition Company $\mathrm{C}$ managed to create an "analysis distributor" which can provide necessary analysis tools for users based on their needs.

\section{Discussion}

Our results show that data combination can provide opportunities to create additional value for customers as well as the company itself. This is true with using both shared datasets from collaboration partners and open, publicly-accessible data sets. At the same time, data processing and cleaning processes are the kind of expertise that makes the company stand out from competitors.

The companies participating in the open IoT platform pilot expressed it, indeed, introduced a beneficial way to improve their expertise, build new applications and services from the 
data, and provide services together with the public partners (especially the city and/or municipals) that cannot be produced alone with limited resources and without collaboration. However, having a "middleman partner" for the data transfer was considered challenging, even if it's benefits for organisation and management were clear.

Taking into account all the tech companies participating to our pilot were already somehow familiar working with the IoT platforms, technical issues for data integration were considered surprisingly low. Issues related to the data size, storing, and easiness of available analysis tools might be considered widely subjective and their challenging related to the company's previous experience. However, questions were raised about the lifetime of open-source projects. If the IoT platform is open source, are there enough developers to keep the platform running for several years and evolve it continuously.

Some of our lessons learned show that when running pilot studies, it is crucial that the company who will be using the data should be part of the sensor selection process and verifying that the data is in correct type. Most of the technical challenges we met were due to the miscommunication or missing information between the pilot partners, city, stakeholders installing the sensors and actual hardware, and companies. Thus, we underline the importance of communication and overall mitigation plans for human-driven errors and misunderstandings.

The monetary issues of relatively small companies participating in open and free IoT platform, raised some open questions and challenges. Solution for aforesaid could be a situation where data is initially free but if company or organization manages to build a service which they sell to customers, company/organization would have to start paying from data. Especially the maintenance and the administration costs were worrying for the small companies and they relied on the municipal partner to cover them. Indeed, continuation of the operation with the open IoT platform cannot be guaranteed after the pilot phase is performed, if there is no common driver and management partner also covering the management costs.

Based on our work, it is clear that the SME companies are willing to use open IoT platforms and especially benefit new and free data sources for their business and products. However, the same companies are not that willing to hand over their own valuable data without getting something in return. This is where the situation becomes more complicated. Data ownership-related questions and intellectual property rights has to be very clearly placed and discussed in such sort of open data-sharing projects. Value of sharing and re-using the company-produced data has to be very clear for all the partners participating in the platform.

\section{CONCLUSION}

In this paper we present the lessons learned and interviewbased analysis of four small or medium-sized companies participating in a open IoT pilot. We focused on the value that utilizing open IoT platform can create for SMEs especially, showing that even if open for utilizing public datasets, companies feel important to have a concrete benefits for sharing their own data.
These include but are not limited to collecting user experience, novel business ideas, and seeing the value created when the data is processed and analyzed instead of in the actual raw data itself. During the pilots, we learned the importance of communication and mitigating human errors, technical challenges being actually the easiest to solve. Communication and planning are especially crucial when making decisions related to the technological solutions, sensor instrumentation, and data formulation.

\section{ACKNOWLEDGMENT}

This work is supported by 6Aika: Future operator independent data integration platform (CityIoT, project A73139).

\section{REFERENCES}

[1] M. Andersen-Gott, G. Ghinea, and B. Bygstad. "Why do commercial companies contribute to open source software?" In: International journal of information management 32.2 (2012).

[2] F. Berkers et al. "Constructing a multi-sided business model for a smart horizontal IoT service platform". In: 17th International Conference on Intelligence in Next Generation Networks (ICIN). IEEE. 2013.

[3] A. Bröring et al. "Enabling IoT ecosystems through platform interoperability". In: IEEE software 34.1 (2017).

[4] Gronroos C. and Voima P. "Critical service logic: making sense of value creation and co-creation". In: Journal of the Academy of Marketing Science 41.2 (2012).

[5] Growth Enabler. Market Pulse Report Internet of Things (IoT). URL: https://growthenabler.com/flipbook/pdf/ IOT\%20Report.pdf. (accessed: 26.09.2019).

[6] FIWARE Foundation e.V. Fiware: The open source platform for our smart digital future. URL: https:// www.fiware.org. (accessed: 02.08.2019).

[7] Foong K.-Y. "Market Trends: Top Opportunities for CSPs in the Internet of Things, 2018-2019". In: Gartner Research (2017).

[8] Golafshani N. "Understanding Reliability and Validity in Qualitative Research". In: The Qualitative Report 8.4 (2003).

[9] European Union Publications Office. Micro-, small- and medium-sized enterprises: definition and scope. URL: https://eur-lex.europa.eu/legal-content/EN/TXT/?uri= LEGISSUM:n26026. (accessed: 30.07.2019).

[10] E. Ries. The lean startup: How today's entrepreneurs use continuous innovation to create radically successful businesses. Crown Books, 2011.

[11] V. Scuotto, A. Ferraris, and S. Bresciani. "Internet of Things: Applications and challenges in smart cities: a case study of IBM smart city projects". In: Business Process Management Journal 22.2 (2016).

[12] E. M. Silva and P. Maló. "IoT testbed business model". In: Advances in Internet of Things 4.04 (2014).

[13] S. Soursos et al. "Towards the cross-domain interoperability of IoT platforms". In: 2016 European conference on networks and communications (EuCNC). IEEE. 2016. 